\title{
Monitoreo de los procesos de educación ambiental: propuesta de estructuración de un sistema de indicadores de educación ambiental*
}

Monitoring Environmental Education Processes:

A Proposal for Structuring a System of

Environmental Education Indicators

Monitoramento dos processos de educação ambiental: proposta de estruturação de um sistema de indicadores de educação ambiental
Alba Nubia Muñoz-Montilla**
iD orcid.org/0000-0003-1486-1410 Pablo Páramo-Bernal ${ }^{* * *}$
iD orcid.org/0000-0002-4551-3040

\begin{abstract}
Artículo de investigación
Revista Colombiana de Educación, N.7 74. Primer semestre de 2018, Bogotá, Colombia.

Para citar este artículo: Muñoz, A. y Páramo, P. (2018). Monitoreo de los procesos de educación ambiental: propuesta de estructuración de un sistema de indicadores de educación ambiental. Revista Colombiana de Educación, (74), 81-106
\end{abstract}

\section{(ब) $(1) \Theta$}

Recibido: 18/12/2016

Evaluado: 18/04/2017

* El presente artículo forma parte de la investigación conducente a la tesis doctoral del Doctorado Interinstitucional en Educación de la Universidad Pedagógica Nacional de la primera autora.

** Magister en Gestión Ambiental y candidata a Doctor en Educación. Docente investigadora Secretaría de Educación del Distrito. Bogotá, Colombia. Correo electrónico: nubiamm@gmail.com

*** Doctor en Psicología. Profesor titular, programa Doctorado Interinstitucional en Educación. Bogotá, Colombia. Correo electrónico: pparamo@pedagogica.edu.co 


\section{Resumen}

A pesar de importantes avances en los últimos años en el desarrollo de políticas de educación en materia ambiental, es poco lo que se sabe del impacto y la efectividad del sinnúmero de actividades, proyectos e iniciativas derivadas de estas políticas en torno a la conservación de los recursos ecológicos y principalmente sobre la formación de las personas en conductas proambientales. En el artículo se formula un sistema de indicadores de educación ambiental, inexistentes de manera formal en Colombia, con la intención de monitorear los alcances y el desarrollo de las acciones educativas que se promueven y emprenden desde distintas políticas y programas institucionales. El sistema propuesto incluye pautas operativas para la recolección de información de los procesos de educación ambiental, en torno a comportamientos ambientalmente relevantes. Con esto se espera contribuir a gestionar y proyectar la educación ambienta en sus distintos niveles de actuación y consolidar conocimiento alrededor de aquellas acciones que resultan más eficaces.

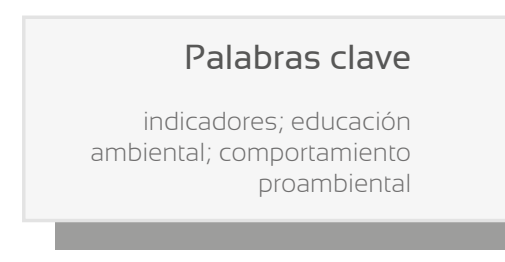

\section{Keywords}

indicators; environmental education; pro-environmental behavior

\begin{abstract}
Despite the significant progress made in the development of environmental education policies in recent years, little is known about the impact and effectiveness of the countless activities, projects and initiatives resulting from those policies on the conservation of ecological resources and, most of all, on people's training in pro-environmental behavior. In this paper, we formulate a system of environmental education indicators, which do not formally exist in Colombia, with the purpose of monitoring the scope and development of the educational actions promoted and undertaken from different policies and institutional programs. The proposed system includes a number of operational guidelines for collecting information from environmental education processes regarding environmentally relevant behaviors. This is expected to contribute to the management and projection of environmental education in the different action levels and to consolidate the existing knowledge on the most effective actions.
\end{abstract}

\section{Resumo}

Apesar de importantes avanços no desenvolvimento de políticas de educação em matéria ambiental nos últimos anos, pouco se sabe do impacto e da efetividade do sem-número de atividades, projetos e iniciativas derivadas dessas políticas sobre a conservação dos recursos ecológicos e principalmente sobre a formação das pessoas em condutas pró-ambientais. No artigo é apresentado um sistema de indicadores de educação ambiental inexistentes formalmente na Colômbia, com a intenção de monitorar a dimensão e o desenvolvimento das ações educativas promovidas e empreendidas desde diversas políticas e programas institucionais. O sistema proposto inclui pautas operativas para a recolecção de informação dos processos de educação ambiental, em torno a comportamentos ambientalmente relevantes. Isso visa contribuir para a gestão e a projeção da educação ambienta nos seus diversos níveis de atuação e consolidar o conhecimento sobre aquelas ações que são mais eficazes.

\section{Palavras-chave}

indicadores; educação ambiental; comportamento pró-ambiental 


\section{Introducción}

Los indicadores ambientales han adquirido relevancia en los últimos años dada su capacidad de generar una imagen sintética de las condiciones ambientales del territorio. Su auge se ha desarrollado de forma paralela a los avances, acuerdos y retos ambientales a nivel global lo que ha orientado la determinación de niveles permisibles, regulaciones y leyes por parte de las entidades encargadas del monitoreo y control ambiental (Suárez, 2003). Gracias a la valoración del estado del ambiente que se ha hecho por medio de estos indicadores, la preocupación por su cuidado ha aumentado, al igual que los esfuerzos en torno a la conceptualización de la educación ambiental, a la construcción de políticas en esta materia y a la implementación de estrategias educativas para llegar a las personas de una manera más efectiva. Los indicadores ambientales también han facilitado las acciones de reconocimiento y comprensión de la dinámica de los ecosistemas estratégicos de la ciudad y la búsqueda de soluciones a las problemáticas que los aquejan. La formulación de la Política Nacional de Educación Ambiental en 2002 y posteriormente el diseño de la Política Pública Distrital de Educación Ambiental en 2007, son una muestra fehaciente del alto grado de desarrollo conceptual en esta materia y de los altos índices de participación ciudadana e institucional para la gestión ambiental en el país.

A pesar de estos importantes avances en materia de política ambiental, es poco lo que se sabe del impacto y la efectividad del sinnúmero de actividades, proyectos e iniciativas de educación ambiental adelantadas desde diferentes frentes (la escuela, las organizaciones sociales, el sector gubernamental) en torno a la conservación de los recursos ecológicos, la defensa del patrimonio ambiental del país y principalmente sobre su eficacia para la formación de las personas en aspectos de preservación ambiental o uso sostenible de los recursos (Páramo, 2009). Al respecto, la Organización de las Naciones Unidas para la Educación, la Ciencia y la Cultura, unESCO (2011) plantea la carencia de criterios unificados que permitan medir la efectividad de los programas y políticas de educación ambiental. En este último aspecto, hay una falencia de estudios que reporten la efectividad y el impacto de la implementación de programas, proyectos y demás estrategias de la educación ambiental no solo en Colombia sino en América Latina; el estudio bibliométrico de Medina y Páramo (2014) da cuenta de ello.

De igual manera, Wilches (2008) plantea como uno de los retos de la educación ambiental la identificación y aplicación de indicadores que faciliten "leer" los procesos educativos y evaluar el impacto de su acción. 
En el mismo sentido, Torres (2012) expone la importancia de adelantar investigaciones que incorporen criterios de sistematización y evaluación permanente de las experiencias educativo-ambientales que posibiliten la retroalimentación y cualificación de estos procesos y viabilicen la participación para la toma de decisiones responsables, en lo relacionado con el manejo del ambiente y el empoderamiento de actores y de escenarios de la educación ambiental.

Pero el problema no radica únicamente en la falta de investigaciones que den cuenta de la efectividad de los programas o proyectos de educación ambiental. Si bien esta evidencia es necesaria, se requiere la formulación de sistemas de indicadores de educación ambiental, inexistentes de manera formal en Colombia. Para ello se hace necesario desarrollar una propuesta que permita dimensionar los alcances de las acciones educativas que se promueven y emprenden desde distintas políticas y programas institucionales y las acciones educativas que se desarrollan desde la escuela y fuera de ella, con el fin de proyectar una gestión más eficiente de las mismas.

Con este fin, en este artículo se explora la existencia de indicadores ambientales y, en particular, indicadores de educación ambiental, en la literatura científica y en la política ambiental nacional e internacional, para posteriormente proponer una estructura conceptual que cobije indicadores en diferentes categorías de información para aplicarla en la construcción de un sistema de indicadores de educación ambiental aplicable al contexto nacional colombiano. Se parte del supuesto de que la elaboración de una propuesta que integre indicadores de los programas e intervenciones de educación ambiental proveería información relevante para tenerla en cuenta en la planeación y proyección del horizonte de acción y facilitaría la implementación de políticas más eficaces siguiendo las tesis de la pedagogía basada en evidencia (Hederich, Martínez y Rincón, 2014; Páramo, en prensa; Páramo y Hederich, 2014).

\section{Panorama del desarrollo de los indicadores ambientales}

Los indicadores ambientales surgieron con el fin de contar con datos concretos de la valoración y evaluación ambiental sobre el estado del ambiente y hacer seguimiento a las políticas que se acordaron en los compromisos internacionales para la protección del medioambiente. Han adquirido relevancia en los últimos años dada su capacidad de generar una imagen sintética de las condiciones ambientales del territorio (Manteiga, 2000). Su auge se ha desarrollado de forma paralela a los avances, acuerdos y retos ambientales en el mundo (Suárez, 2003). 
Al revisar el panorama de los indicadores ambientales a nivel nacional e internacional, se encuentra concordancia entre el avance en la agenda ambiental y de desarrollo sostenible en el mundo y el alto desarrollo conceptual y metodológico de los indicadores. Este interés es sin lugar a dudas producto de la creciente preocupación por la valoración del estado de los recursos naturales y por la medición de los impactos ambientales sobre los recursos naturales, manifestada por la comunidad mundial desde la Declaración de Estocolmo en 1972, ratificada en la Agenda 21 de la Declaración de Río de Janeiro en 1992 y en la creación de la Comisión para el Desarrollo Sostenible, en 1996, que generó un conjunto de indicadores de desarrollo sostenible y el desarrollo de hojas metodológicas para su medición (Comisión Económica para América Latina y el Caribe, Cepal, 2007).

A partir de su origen y conceptualización en el tiempo, se han agrupado en: indicadores de primera generación, que se enfocan en aspectos de la dinámica ambiental y en indicadores de segunda generación o de desarrollo sostenible (Quiroga, 2007; Rey, 2002). También se ha avanzado en el diseño e implementación de indicadores de sostenibilidad, que vinculan los aspectos económico, social y ambiental en forma transversal y sistemática (Cepal, 2007). Otro impulso importante en el tema de indicadores lo constituye la producción de superíndices o megaindicadores agregados de importancia internacional, como son la huella ecológica, el índice del planeta vivo y el índice de sostenibilidad ambiental.

\section{Indicadores ambientales en Colombia}

En el país se han adelantado varias iniciativas de indicadores ambientales y de desarrollo sostenible a partir de las cuales se han llevado a cabo numerosos ejercicios de formulación de sistemas de información e indicadores ambientales y de gestión a lo largo de las últimas décadas. La primera experiencia, en 1996, fue liderada por la Unidad de Política Ambiental (UPA) del Departamento Nacional de Planeación (DNP), en convenio con el Centro Internacional de Agricultura Tropical (CIAT). La metodología utilizada correspondió al marco ordenador presión-estado-respuesta, al cual le fueron incorporadas las categorías de efecto/impacto y gestión (Cepal, 2007).

En 1998 se produjo un nuevo impulso a la generación de indicadores ambientales, a través de la formulación de la Política Nacional Ambiental. Se inició la labor interinstitucional para la creación de un Sistema Nacional de Indicadores Ambientales que respondiera a las necesidades de información ambiental estratégica del país y que en 2002 orientó la publicación de los Indicadores de la línea base del sistema de Información Ambiental de Colombia (Ministerio de Ambiente, Vivienda y Desarrollo Territorial [MAVDT], 2007). 
El Sistema de Información Ambiental para Colombia (SIAC) procesa datos e información sobre el estado (cantidad y calidad), uso y aprovechamiento, vulnerabilidad y sostenibilidad del ambiente y recursos como: agua, biodiversidad, suelo y aire. Según el Decreto 1200 de 2004, el SIAC está formado por dos subsistemas: El Sistema de Información Ambiental (SIA), para el seguimiento a la calidad y el estado de los recursos naturales y el ambiente, y el Sistema de Información para la Planeación y Gestión Ambiental (SIPGA). El SIA se apoya en el Sistema Nacional de Indicadores Ambientales. Los indicadores en su mayoría son generados por las entidades que componen el Sistema Nacional Ambiental (SINA). Los lineamientos de los indicadores los establece el Grupo de Gestión de Indicadores y Monitoreo Ambiental del SIA, conformado por representantes del Ministerio de Ambiente y Desarrollo Sostenible (MADS) y de los cinco institutos de investigación ambiental del país, con el acompañamiento del Departamento Administrativo Nacional de Estadística (DANE) y del Departamento Nacional de Planeación (DNP) (MAVDT, 2007).

\section{Indicadores ambientales en el Distrito Capital}

De manera particular, se reseñan a continuación los mecanismos de seguimiento de las condiciones ambientales a través de indicadores que se adelantan en Bogotá, Distrito Capital, por el mayor grado de desarrollo que ha tenido el tema en esta ciudad.

Los indicadores ambientales en el Distrito Capital han sido promovidos mediante el Decreto Distrital 681 de 2011, a través del cual se adopta el Observatorio Ambiental de Bogotá (OAB) y el Acuerdo Distrital 67 de 2002, que establece que la Administración Distrital deberá hacer público el estado y la evolución de los indicadores de gestión de la Administración Distrital.

Por el Decreto Distrital 681 del 30 de abril de 2011 se crea y adopta el Observatorio Ambiental de Bogotá como sistema oficial de reporte y divulgación de la información, las estadísticas y los indicadores ambientales que producen las entidades de la Administración Pública Distrital.

El OAB es un sitio web que permite conocer a través de indicadores ambientales el estado y la calidad del ambiente en Bogotá, así como los resultados de la gestión desarrollada por varias entidades del Sistema de Indicadores de Gestión Ambiental del Distrito Capital (SIGA) (SDA, 2015). En la actualidad, el OAB está conformado por 482 indicadores, organizados por recurso natural (256) y por tema (279) (véase la tabla 1). 
Tabla 1. Indicadores Observatorio Ambiental de Bogotá

\begin{tabular}{|c|c|c|}
\hline & Recurso/Tema & $\begin{array}{c}\text { No. de } \\
\text { indicadores }\end{array}$ \\
\hline \multirow{7}{*}{$\begin{array}{l}\text { Indicadores por } \\
\text { recurso natural }\end{array}$} & Agua & 64 \\
\hline & Aire & 19 \\
\hline & Calidad del aire & 18 \\
\hline & Ruido & 5 \\
\hline & Fauna & 2 \\
\hline & Suelo & 10 \\
\hline & Vegetación & 168 \\
\hline \multirow{8}{*}{ Indicadores por tema } & Cambio climático & 43 \\
\hline & Control para la calidad ambiental & 124 \\
\hline & Ecosistemas & 10 \\
\hline & Gestión ambiental empresarial & 17 \\
\hline & Movilidad Sostenible & 19 \\
\hline & Educación ambiental y participación & 2 \\
\hline & Salud ambiental & 14 \\
\hline & Ordenamiento y ecourbanismo & 190 \\
\hline \multicolumn{2}{|c|}{$\begin{array}{c}\text { Indicadores del Plan de Acción Cuatrienal Ambiental de } \\
\text { Distrital Capital, PACA (2012-2016) }\end{array}$} & 26 \\
\hline
\end{tabular}

Fuente: Elaboración propia a partir de información del Observatorio Ambiental de Bogotá (http://oab. ambientebogota.gov.co/)

En cuanto a los indicadores de gestión de las entidades de la Administración Distrital, el Acuerdo 67 de septiembre 9 de 2002 del Concejo de Bogotá establece que esta deberá hacer público el estado y la evolución de los indicadores de gestión en la administración distrital en diversos aspectos. En el artículo cuarto, se determina que la Secretaría General de la Alcaldía Mayor de Bogotá diseñará las metodologías, los procedimientos y mecanismos necesarios para la recolección de la información y publicación de los indicadores establecidos en el Acuerdo, en el portal de internet de la Alcaldía Mayor en un enlace bajo el título: Sistema de Indicadores de Gestión y será actualizada semestralmente con corte a 30 de junio y 31 de diciembre de cada año (http://www.alcaldiabogota.gov. co/sisjur/normas/Norma1.jsp?i=5461). 


\section{Panorama de los indicadores de educación ambiental en el plano internacional y nacional}

Como se ha hecho notar, a pesar del amplio desarrollo que han tenido los indicadores ambientales en los últimos años, es poco lo que se ha generado hasta la fecha en torno a la medición de la efectividad y el impacto de la educación ambiental, que se constituye en el pilar de la sostenibilidad y de la protección ambiental y de la instauración de prácticas armónicas del ser humano en el territorio.

Se evidencia la carencia de mecanismos de valoración de las estrategias, acciones y programas de educación ambiental que se adelantan día a día en diversas instancias del orden local, distrital y nacional. De ahí la importancia de definir indicadores que proporcionen una visión de la situación en la que se encuentra esta disciplina, las dificultades que afronta, los aciertos y potencialidades que permiten establecer líneas de actuación que posibiliten un mejor desarrollo de la formación educativa ambiental de los ciudadanos.

Al explorar el desarrollo de indicadores de educación ambiental en los planos nacional e internacional, no es tan prolífico como se esperaría. En la revisión documental realizada a través de bases electrónicas de búsqueda se hallaron algunas propuestas de indicadores enfocadas en la evaluación de aspectos puntuales del desarrollo de la educación ambiental. Se encontraron, como se muestra a continuación, formulaciones para la evaluación de programas de educación ambiental y evaluación de la gestión ambiental municipal en España; una propuesta para la evaluación de la educación ambiental en las áreas protegidas del Parque Nacional de Triglav de Eslovenia; un modelo para la evaluación de la educación, la interpretación y la información ambiental en espacios naturales protegidos (ENP) de las islas Baleares; una propuesta de criterios e indicadores para la evaluación de la funcionalidad de los sistemas regionales para la educación ambiental en Italia y una guía de la unESCO para la evaluación de la educación ambiental en las escuelas.

A continuación se presenta una síntesis de estas propuestas y se resaltan elementos de algunas de ellas, que se consideran pertinentes para la estructuración del sistema de indicadores de educación ambiental en Colombia, objeto del presente artículo. 


\section{Propuesta de indicadores de desarrollo de la educación ambiental en España}

De Esteban, Benayas y Gutiérrez (2000) y De Esteban (2002) presentan una propuesta de indicadores de desarrollo de educación ambiental que busca evaluar la efectividad y el impacto de los programas en esta área y estructurar actuaciones para el desarrollo de una educación ambiental de calidad en España. Estos autores identifican tres grupos de variables:

1. Indicadores de medios y recursos en educación ambiental, a través de los cuales se hacen estimaciones de los gastos en educación ambiental por parte de la administración ambiental tanto para equipamientos ambientales, como para la realización de jornadas sobre esta disciplina. Los indicadores que incluye De Esteban (2002) son: total de gasto en educación ambiental por habitante, número de participantes en las jornadas por cada mil habitantes, número de equipamientos ambientales por cada mil habitantes, jornadas celebradas en educación ambiental por cada mil habitantes y número de tesis doctorales sobre educación ambiental.

2. Indicadores de gestión ambiental, que engloban acciones negativas o positivas por parte de los ciudadanos hacia el ambiente. Los que incluye De Esteban (2002) son: porcentaje de superficie incendiada, número de agresiones hacia el medioambiente, el reciclaje de vidrio y el porcentaje de superficie protegida sobre el total.

3. Indicadores de actitudes y motivaciones ambientales de los ciudadanos, que reflejan las opiniones, predisposiciones y valoraciones de los ciudadanos hacia el ambiente. Las actitudes de los individuos se evalúan mediante encuestas de opinión sobre el grado de interés hacia los problemas ambientales. De Esteban (2002) propone como indicadores el porcentaje de ciudadanos que reclama una mayor y más eficiente educación ambiental, los índices de audiencia de un programa de sensibilización ambiental y el número de organizaciones ecologistas locales por cada mil habitantes, entre otros.

De Esteban et al. (2000) proponen la utilización de una ficha para cada indicador, que contenga datos relacionados con la denominación del indicador, su definición, su unidad de medida, su pertinencia para el adecuado desarrollo de la educación ambiental, la fuente, su relación con otros indicadores, su evolución en el tiempo y sus alcances y posibles sesgos y limitaciones. 
Guía de evaluación de la educación ambiental en las escuelas para América Latina y el Caribe

Esta guía, publicada por la UNESCO, tiene el propósito de medir la efectividad y eficiencia de los programas de educación ambiental que se adelantan en las escuelas. Efectividad en términos de los efectos y resultados obtenidos, y eficiencia en cuanto a que los métodos utilizados y las acciones realizadas sean los mejores en términos de costos, tiempo requerido y adecuación al trabajo (Bennet, 1993).

Para medir la efectividad y eficiencia del programa de educación ambiental, Bennet (1993) plantea la evaluación de tres aspectos:

» Evaluación de las aptitudes de pensamiento crítico, referidas a la aplicación de conocimientos, análisis de objetos y eventos, síntesis como capacidad de reunir elementos en un todo unificado y evaluación de objetos y eventos

» Progreso en el aprendizaje de los alumnos

» Habilidades de acción, a partir de seis categorías de acción ambiental establecidas por Hungerford y Litherland (1986): persuasión, consumismo, acción política, acción legal, ecogestión e interacción.

Modelo para la evaluación de la educación, la interpretación y la información ambiental en ENP de las islas Baleares

El modelo de Sureda, Oliver y Castells (2004) reúne indicadores relativos a la planificación de las tareas educativas, interpretativas e informativas en relación con: acciones dirigidas a la población local; acciones dirigidas al público en general; existencia de equipamientos, recursos humanos y recursos económicos dedicados a educación ambiental; características de los itinerarios, de los centros de visitantes y de las señalizaciones; y accesibilidad.

\section{Propuesta de indicadores de educación ambiental del Parque Nacional de Triglav (TNP) de Eslovenia}

La propuesta del Parque de Triglav (Stubelj, 2013) presenta una lista de 89 indicadores de educación ambiental agrupados en 14 dimensiones para evaluar las prioridades en la aplicación de la educación ambiental en las áreas protegidas. Entre estas dimensiones sobresalen: Actividades directas de educación ambiental realizadas en el Parque Triglav (15 indicadores), publicaciones (12 indicadores), eventos ( 3 indicadores), proyectos (3 indicadores), cursos (2 indicadores), visitantes (10 indicadores), entre otros. La importancia de los indicadores se midió en una escala de tres valoraciones: esencial $=3$, deseable $=2$, no significativa $=1$. 


\section{Propuesta de indicadores de educación ambiental en municipios de España}

García y Benayas (2007) diseñaron una propuesta de indicadores de educación ambiental relacionados con indicadores de sostenibilidad a escala municipal, por considerar el ámbito local como escala de trabajo idónea para el estudio de las relaciones entre el ser humano y su ambiente desde un punto de vista multidisciplinar. En este sentido, Heras y Sintes (2004) consideran que el desarrollo de la educación ambiental a nivel local no ha ido a la par del desarrollo en su evaluación, tal y como muestran los pocos estudios e indicadores que, para esta área, se han desarrollado en ámbitos locales.

De manera complementaria, Ramírez (2002), al evaluar distintos sistemas de indicadores locales de sostenibilidad existentes en la actualidad, plantea que muchos indicadores se centran en aspectos técnicos directos como el tratamiento de residuos, la depuración de aguas residuales o la contaminación atmosférica y que son todavía pocos los estudios que indaguen acerca de las relaciones entre las conductas ambientales de una población determinada y sus características socioeconómicas, o acerca de la percepción que tiene la población de los problemas ambientales.

Para el diseño de indicadores de educación ambiental, García y Benayas (2007) seleccionaron variables que pudieran convertirse en indicadores. Para ello, atendieron a dos criterios: por un lado, la representatividad de las variables con relación a aspectos de la educación ambiental municipal, como gestión, diversidad de recursos, diversidad de temáticas, trayectoria del ayuntamiento en proyectos, participación, diversidad de técnicas de comunicación; por otro, la facilidad de obtención de información de las variables, que posibilitara el seguimiento por parte de técnicos municipales y evaluadores. Entre las variables seleccionadas por estos autores se destacan: gasto municipal por habitante, diversidad de recursos, diversidad de temáticas, destinatarios, técnicas de comunicación, índice de participación e índice de coherencia de las actuaciones de educación ambiental en función de los problemas ambientales percibidos. A partir de estas variables, García y Benayas (2007) infirieron que los factores subyacentes que determinan, en mayor o menor medida, el grado de desarrollo de la educación ambiental en los ayuntamientos de la muestra son:

» Factor I: el diseño de actuaciones de educación ambiental

» Factor II: la consolidación de los procesos de educación ambiental

» Factor III: los recursos económicos destinados a promover la participación (García y Benayas, 2007). 
Teniendo en cuenta las variables asociadas a cada factor, concluyeron que el más importante a la hora de determinar el grado de desarrollo de la educación ambiental es el diseño de las actuaciones en esta materia.

Sistema de evaluación de la funcionalidad de los sistemas regionales para la educación ambiental en Italia

En el 2004 se realizó un estudio en Italia sobre la calidad de los sistemas regionales para la educación ambiental, a partir del cual se propuso un sistema de evaluación coherente y flexible que permite aplicar dentro de cada región italiana los mismos criterios a cualquier proyecto o sujeto promotor, y a la vez, generar una reflexión con los funcionarios regionales respecto de la calidad de la educación ambiental (Mayer, 2006).

La propuesta de Mayer (2006) se basa en seis criterios estrechamente relacionados con las funciones del sistema regional para la educación ambiental: la educación, orientada a la formación para la ciudadanía; la formación para una cultura ambiental; la comunicación y la información orientadas a la educación ambiental; el apoyo a los procesos de desarrollo local, la coordinación interinstitucional y la evaluación y la investigación social vinculada a los procesos de desarrollo local sostenible.

\section{Sistema Nacional de Indicadores Educativos para los niveles de preescolar, básica y media en Colombia}

En Colombia no existe una formulación de indicadores que permita evaluar la efectividad o el impacto de las intervenciones y los procesos desarrollados en educación ambiental. Sin embargo, el Ministerio de Educación Nacional formuló en 2013 un Sistema Nacional de Indicadores Educativos, que a pesar de no estar dirigidos directamente a la educación ambiental establecen categorías que los autores del presente artículo consideran pertinentes para la evaluación y el monitoreo de los procesos e intervenciones de educación ambiental.

El sistema de indicadores del MEN (2013) contempla cinco componentes enmarcados en el enfoque sistémico y sus relaciones:

» los contextuales, que hacen referencia al entorno demográfico, social y económico en el cual se desenvuelve la educación

» los recursos que se invierten en el sistema educativo

» el proceso o funcionamiento del sistema educativo

» los resultados del sistema

» el impacto generado por la educación sobre el mismo entorno 
Asimismo, el documento incluye el sistema con base en el cual se toma la información para el cálculo de los indicadores y una selección de indicadores por cada componente.

En el plano distrital, al indagar en el tema de educación ambiental y participación del OAB se encuentran dos indicadores: Personas vinculadas a Estrategias de Educación Ambiental (Estedam) y Población Sensibilizada con el Programa Basura Cero (PSPBC) (http://oab.ambientebogota.gov.co/).

El primer indicador tiene como objetivo medir el número de "ciudadanos pertenecientes a organizaciones, universidades, colegios o comunidad general que son beneficiados de las dinámicas de participación y educación ambiental en pro de la sensibilización, apropiación, recuperación y protección del ambiente bogotano" (http://oab.ambientebogota.gov.co/). La información para realizar la medición de este indicador se recoge a partir de la vinculación de la ciudadanía en las estrategias de educación ambiental priorizadas por cada localidad en el marco de la Política Pública Distrital de Educación Ambiental (http://oab.ambientebogota.gov.co/).

El segundo indicador mide el número de personas participantes en procesos de socialización y sensibilización en el Programa Basura Cero. La información para la medición de este indicador se recoge en Suba, una de las localidades de Bogotá, donde se ha fomentado entre sus habitantes una cultura de manejo de los residuos sólidos y de cuidado, preservación y conservación del ambiente (http://oab.ambientebogota.gov.co/).

De los documentos revisados se retoman a continuación elementos que consideramos pertinentes para la propuesta de estructuración de un sistema de indicadores de educación ambiental aplicables a Colombia.

\section{Estructuración de un sistema de indicadores de educación ambiental}

A partir de las fuentes consultadas y en concordancia con los fines de la Política de Educación Ambiental de Colombia, se ha diseñado una propuesta de organización de un sistema de indicadores de educación ambiental que contiene:

$»$ consideraciones conceptuales y metodológicas para el procesamiento de la información y la construcción de indicadores.

» ficha para la documentación de indicadores.

» estructura del sistema de indicadores de educación ambiental.

» propuesta operativa para la gestión de la información requerida para alimentar el sistema de indicadores.

» el enunciado de algunos indicadores para cada categoría. 


\section{Consideraciones conceptuales y metodológicas para el procesamiento de la información y construcción de indicadores}

Para procesar los datos provenientes del levantamiento de información, obtenidos a través de los diversos medios disponibles, se requiere su ordenación, clasificación y la descripción en series de tiempo según sea su disponibilidad y objetivo para producir conjuntos de estadísticas.

El DANE (2012), que como ente coordinador estadístico nacional promueve la capacidad técnica para la organización y producción de información estratégica de calidad, plantea cinco etapas para la construcción de indicadores: (1) identificación del objeto de medición; (2) determinación del aspecto específico que se va a evaluar; (3) elaboración del indicador a partir de la identificación de las variables que lo conforman y de la relación entre ellas; (4) selección de indicadores y calidad de los datos; (5) identificación del contexto, para determinar los usos específicos que tendrá el indicador y señalar los actores que requieren esa información.

Dado que los indicadores deben ser de fácil comprensión e interpretación y deben permitir establecer relaciones con otros indicadores utilizados para medir la situación o fenómeno en estudio, deben ser elaborados de forma sencilla, automática, sistemática y continua. Chacón (2004) define características que deben cumplir los indicadores: Validez, confianza, flexibilidad, sensibilidad, objetividad, mensurabilidad, eficacia y claridad. Leva (2005) propone como criterios generales para la selección de indicadores: representatividad, que sean susceptibles de medición, pertinencia, funcionalidad, disponibilidad, confiabilidad, utilidad y la posibilidad de estandarización del indicador que permita realizar comparaciones en diferentes escenarios. La elección de los indicadores puede realizarse a través de dos métodos: deductivo (cuando surgen de un marco teórico) e inductivo (cuando los indicadores son determinados por la disponibilidad de datos). En general, los sistemas de indicadores combinan ambos métodos (Leva, 2005).

\section{Ficha para la documentación de indicadores}

Para la documentación de los indicadores, se acogen las propuestas del DANE (2012) y De Esteban, Benayas y Gutiérrez (2000), que plantean el diseño de una ficha técnica del indicador. Esta se constituye en un instrumento metodológico de resumen y su contenido está detallado en la tabla 2. 


\begin{tabular}{|c|c|}
\hline $\begin{array}{l}\text { Nombre del } \\
\text { indicador }\end{array}$ & Es la expresión verbal, precisa y concreta que identifica el indicador. \\
\hline Sigla & Término abreviado que representa el nombre del indicador. \\
\hline Objetivo & $\begin{array}{l}\text { Propósito que se persigue con su medición. Describe la naturaleza y } \\
\text { finalidad del indicador. }\end{array}$ \\
\hline $\begin{array}{l}\text { Definiciones y } \\
\text { conceptos }\end{array}$ & $\begin{array}{l}\text { Explicación conceptual de cada uno de los términos utilizados en el } \\
\text { indicador. }\end{array}$ \\
\hline $\begin{array}{l}\text { Método de } \\
\text { medición }\end{array}$ & $\begin{array}{l}\text { Corresponde a la explicación técnica sobre el proceso para la obten- } \\
\text { ción de los datos utilizados y la medición del resultado del indicador. }\end{array}$ \\
\hline $\begin{array}{l}\text { Unidad de } \\
\text { medida }\end{array}$ & Unidad en la que se mide el indicador. \\
\hline Fórmula & $\begin{array}{l}\text { Expresión matemática mediante la cual se calcula el indicador. La } \\
\text { fórmula se debe presentar con siglas claras y que, en lo posible, den } \\
\text { cuenta del nombre de cada variable. }\end{array}$ \\
\hline Variables & $\begin{array}{l}\text { Descripción de cada variable de la fórmula. Especificación precisa de } \\
\text { cada una de las variables con su respectiva sigla. }\end{array}$ \\
\hline $\begin{array}{l}\text { Fuente de los } \\
\text { datos }\end{array}$ & $\begin{array}{l}\text { Nombre de las entidades encargadas de la producción y/o suminis- } \\
\text { tro de la información que se utiliza para la construcción del indicador } \\
\text { y operación estadística que produce la fuente. }\end{array}$ \\
\hline $\begin{array}{l}\text { Periodicidad de } \\
\text { los datos }\end{array}$ & $\begin{array}{l}\text { Frecuencia con que se hace la medición del indicador en su expre- } \\
\text { sión total. }\end{array}$ \\
\hline $\begin{array}{l}\text { Fecha de } \\
\text { información } \\
\text { disponible }\end{array}$ & Se refiere a la fecha inicial y final de la información disponible. \\
\hline Responsable & $\begin{array}{l}\text { Entidad(es) y dependencia(s) que tendrá(n) a su cargo la elaboración } \\
\text { del indicador. }\end{array}$ \\
\hline Observaciones & $\begin{array}{l}\text { Incluye las reflexiones y recomendaciones que se consideren } \\
\text { pertinentes para la conceptualización y comprensión del indicador, } \\
\text { además de señalar la bibliografía de referencia o documental utiliza- } \\
\text { da para la elaboración de los conceptos. }\end{array}$ \\
\hline
\end{tabular}

Fuente: Adaptado de DANE (2012) y De Esteban, Benayas y Gutiérrez (2000)

\section{Estructura del sistema de indicadores de educación ambiental}

A partir de la conceptualización presentada, se considera que la configuración de un sistema de indicadores de educación ambiental para Colombia deberá estar orientada a generar herramientas de monitoreo de la situación actual de la educación ambiental en el país, del estado de los procesos educativos que se han adelantado en esta materia y el impacto de estos procesos tanto para la gestación de cambios comportamentales de los individuos como para el aumento de acciones de mejoramiento ambiental. 
Si bien es cierto que no existe un consenso respecto de lo que debe ser un buen indicador de desarrollo de la educación ambiental (De Esteban, 2002), o de las dimensiones que brinden un panorama de la efectividad de sus acciones y programas, a continuación se presenta una propuesta de estructuración de un sistema de indicadores para la educación ambiental. Esta propuesta integra elementos metodológicos desarrollados por el DANE (2012), elementos conceptuales que aportan las propuestas descritas, integrados con las categorías de información propuestas en el Sistema Nacional de Indicadores Educativos de Colombia (MEN, 2013), relacionadas con el contexto, los recursos, los procesos, logros e impacto de las estrategias implementadas en educación ambiental, con el propósito de dar lugar a un sistema de monitoreo de este tema acorde con los fines de la Política Nacional de Educación Ambiental y con la Política Pública Distrital de Educación Ambiental.

La estructura de este sistema se ha configurado en torno a los comportamientos ambientalmente relevantes (Corral y Pinheiro, 2004), por considerar que integran conductas proambientales y proecológicas de protección del ambiente, promueven el bienestar humano e implican niveles de pensamiento y reflexión mucho más personales.

Corral (2000) define la conducta protectora del ambiente como "el conjunto de acciones intencionales, dirigidas y efectivas que responden a requerimientos y que resultan en la protección del medio". Entre los principales comportamientos ambientalmente relevantes, considerados en el presente artículo, se encuentran: la promoción del consumo responsable, la disposición y el manejo de los residuos, la generación de buenas prácticas alrededor de la movilidad ecológica y la protección de los recursos ecológicos de la ciudad.

La estructura del sistema de indicadores de educación ambiental (véase la figura 1) está cimentada en dos dimensiones, que buscan abarcar de manera integral tanto las diversas categorías de información como la fuente de donde proviene esta. La primera dimensión contempla las categorías de información propuestas en el Sistema Nacional de Indicadores Educativos de Colombia (MEN, 2013), con el propósito de guardar coherencia con la política nacional educativa existente, que brinden información del contexto, los recursos, procesos, logros e impacto de las estrategias implementadas en educación ambiental. La segunda, se refiere a la fuente de donde proviene la información de programas, proyectos, acciones o iniciativas de educación ambiental, por considerar que son varios frentes productores de información al respecto: la escuela, la sociedad civil, el ámbito académico y la gestión gubernamental para la educación ambiental. 


\section{Educación Ambiental}

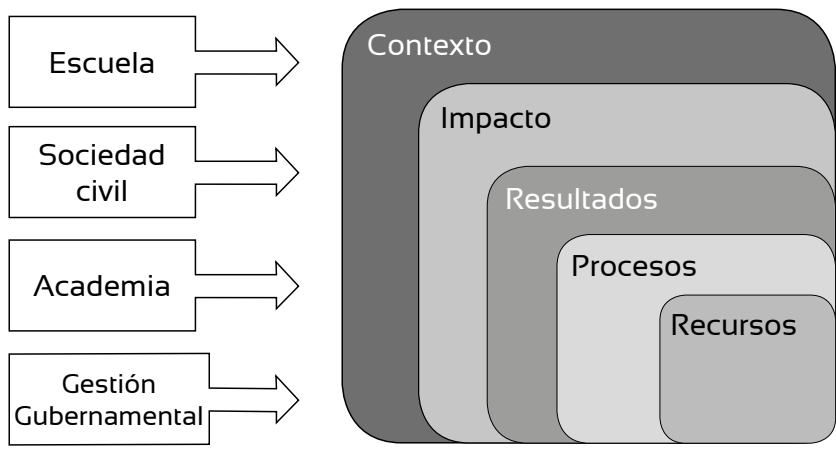

Comportamientos Ambientalmente Relevantes

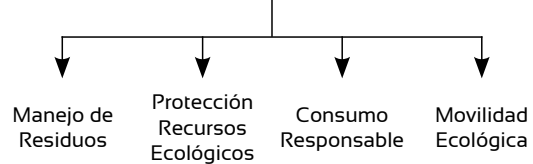

Figura 1. Estructura del Sistema de Indicadores de Educación Ambiental Fuente: elaboración propia

A continuación se definen los componentes del sistema de indicadores. Inicialmente se definen las categorías de indicadores propuestos por el MEN (2013) y adaptados para el monitoreo de la educación ambiental:

» Los indicadores de contexto pueden monitorear la cobertura de los proyectos en los diferentes niveles educativos y la población que está siendo objeto de los procesos de educación ambiental.

» Los indicadores de impacto refieren información relacionada con cambios comportamentales de los individuos y transformaciones de los ambientes como producto de la implementación de acciones, proyectos o programas de educación ambiental. Se propone monitorear el impacto a través de la medición de la huella ecológica, una medida que refleja el consumo de recursos de cada individuo, a través del cálculo personal de consumo de energía eléctrica, agua, generación de residuos y medio de transporte utilizado.

» Los indicadores de resultados permiten observar el cumplimiento de los objetivos propuestos para cada uno de los programas de educación ambiental ejecutados. 
Los indicadores de procesos informan sobre la organización y dinámica de los procesos de formación en términos de conocimiento ambiental y actitudes proambientales como resultado del efecto de la implementación de la educación ambiental.

" Los indicadores de recursos evalúan la cantidad de recursos humanos, financieros y materiales destinados para los programas y proyectos de educación ambiental.

En cuanto a las fuentes de información, estas se refieren a:

» Los proyectos e intervenciones ambientales desde la escuela. Se pueden monitorear mediante la evaluación de iniciativas y proyectos ambientales, planteados desde el ámbito educativo, que involucran tanto procesos de formación ambiental como estrategias de intervención sobre el territorio, a través de prácticas de mejoramiento ambiental, de recuperación ecológica, de transformación de espacios o que promueven el reconocimiento y la comprensión de los elementos estructurantes del territorio y de su dinámica. El ámbito de la escuela es de particular importancia por reunir las condiciones formales para la formación ambiental.

» Las acciones desde la sociedad civil. Son mediciones que se derivan de iniciativas y proyectos ambientales, propuestos desde organizaciones y grupos ambientales que generan acciones de participación de la sociedad civil en torno a procesos de educación ambiental e intervenciones de mejoramiento ambiental.

» La generación de conocimiento ambiental desde el ámbito académico, cuya medición se realiza a través de recuentos y análisis bibliométricos de artículos, publicaciones, informes y diversas estrategias de divulgación en materia de educación ambiental. Incluye además la medición de proyectos de investigación en educación ambiental formulados y ejecutados desde instituciones del ámbito académico, como universidades, centros de investigación o centros de monitoreo ecosistémico, entre otros.

» La gestión gubernamental para la educación ambiental se mide a través del seguimiento a la formulación de políticas, el diseño de programas y la destinación de recursos presupuestales para la educación ambiental, desde las diferentes instancias gubernamentales. También se mide a partir de la cantidad y características de los equipamientos ambientales. 


\section{Propuesta operativa para la gestión de la información del Sistema de Indicadores de Educación Ambiental}

Previo a la formulación de indicadores, se requiere identificar las fuentes de información, establecer los mecanismos para su recolección y determinar los procedimientos para su centralización y procesamiento estadístico.

El punto de partida se orienta con preguntas como: ¿Qué instituciones poseen información del tema? ¿La información está disponible? ¿Dónde se producen los datos? ¿En qué formato se encuentran? ¿Qué métodos utilizaría para recolectar la información? ¿Cómo se captan o recolectan los datos? ¿Cómo fluyen?

El DANE (2012) sugiere elaborar un directorio de fuentes, donde se describan claramente los datos de identificación de cada una y la información disponible.

Dado que la información de educación ambiental puede provenir de espacios de educación formal e informal, del ámbito gubernamental, de la sociedad civil y del ámbito académico e incluye para su recolección aportes de varias instituciones, se recomienda la centralización a través de un observatorio de educación ambiental, concentrado en una institución universitaria de carácter público.

Para el manejo y recopilación de información se propone el siguiente procedimiento a partir de la fuente de donde provienen los datos (véase la figura 2):

» La recopilación de información relacionada con proyectos ambientales escolares (Prae) y convenios interinstitucionales de apoyo a la formulación y acompañamiento a Prae puede valerse de: (1) El portal de internet que posee actualmente el Ministerio de Educación Nacional, en http://aplicaciones.colombiaaprende.edu.co/prae/, a través del enlace: Instituciones Educativas registradas en la Ficha Prae o en el Edusitio de educación ambiental de la página web del men; (2) el portal de la Secretaría de Educación Distrital, a través del diligenciamiento de fichas de Prae, que se adelantan en el Distrito Capital, al correo educacionambientalsed@gmail.com; (3) el portal de la Secretaría Distrital de Ambiente, en http://www. ambientebogota.gov.co/; y (4) la página web de las secretarías de educación municipales.

» La información de proyectos e iniciativas de la sociedad civil, proveniente de Proyectos Ciudadanos de Educación Ambiental (Procedas) o proyectos ejecutados por organizaciones no gubernamentales ambientalistas, debidamente inscritas ante la Secretaría 
Distrital de Ambiente, se registrará en la página web de esta institución, en http://www.ambientebogota.gov.co/ en el caso de las instituciones del orden distrital y será reportada ante el Ministerio de Ambiente y Desarrollo Sostenible, en el caso de organizaciones del orden departamental y/o nacional.

»Los proyectos e iniciativas adelantadas por colectivos ambientales de carácter barrial y local reportarán los resultados de sus actividades a través de las alcaldías locales, quienes a su vez los incorporarán a los sistemas de información de la Secretaría Distrital de Ambiente.

» La información de la gestión gubernamental para la educación ambiental, en lo respectivo a formulación de políticas y destinación de recursos para la educación ambiental, deberá reportarse desde la entidad ejecutora, a las alcaldías locales, quienes a su vez, dentro de su función de fortalecimiento de la gestión pública local, reportarán a la Secretaría de Gobierno Distrital, en http:// www.gobiernobogota.gov.co/, o a la Secretaría General de la Alcaldía Mayor, en el caso de las instituciones del orden distrital y al Ministerio de Ambiente y Desarrollo Sostenible, si se trata de instituciones del orden departamental y/o nacional, para que pueda ser incorporada de acuerdo al sistema de indicadores de gestión y reportada semestralmente por estas dependencias.

» Los resultados de la ejecución de programas y convenios interinstitucionales serán informados al Comité Interinstitucional de Educación Ambiental Cidea, que se reúne periódicamente.

» La información proveniente de proyectos y convenios ejecutados por grupos académicos, grupos de investigación y/o universidades será reportada a la Red Académica de Educación Ambiental, liderada por el IDEA de la Universidad Nacional y de la cual forman parte representantes de varias universidades.

» La medición de la generación de conocimiento de educación ambiental proveniente del ámbito académico, a través de publicaciones de artículos, revistas y libros se centralizará directamente en el observatorio de educación ambiental para realizar los análisis bibliométricos respectivos. 


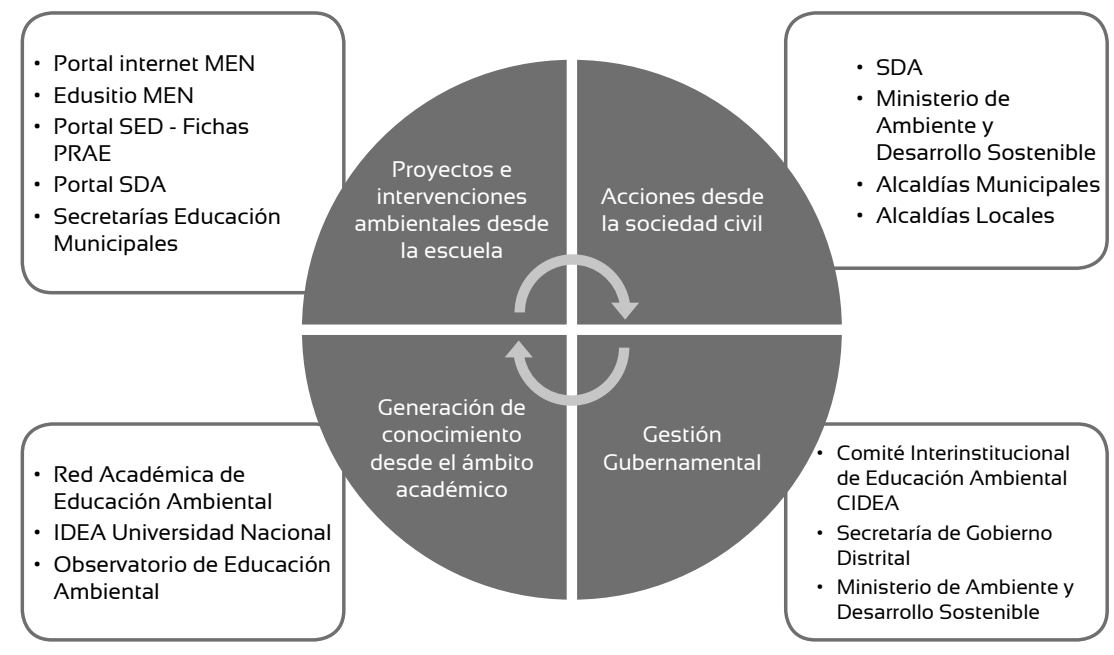

Figura 2. Gestión de la información en el Sistema de Indicadores de Educación Ambiental Fuente: elaboración propia

\section{Propuesta de indicadores para cada categoría}

Finalmente, a partir de los elementos anteriores, se enuncian indicadores para cada categoría de información que deberían integrarla. El organismo encargado de hacer el seguimiento a estos indicadores podrá valerse de la siguiente guía para señalar la fuente de información.

Tabla 3. Propuesta de indicadores para cada categoría

\begin{tabular}{|c|c|c|c|c|c|}
\hline \multirow[b]{2}{*}{ Categoría } & \multirow[b]{2}{*}{ Indicadores } & \multicolumn{4}{|c|}{ Fuente de información } \\
\hline & & $\begin{array}{l}\frac{\pi}{U} \\
己 \\
\tilde{U} \\
\tilde{U}\end{array}$ & 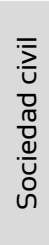 & 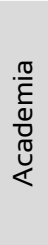 & 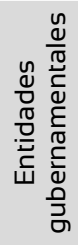 \\
\hline Contexto & $\begin{array}{l}\text { Cobertura de los proyectos de EA por grupo de edad } \\
\text { Proporción de la población que ha participado en } \\
\text { procesos de educación ambiental } \\
\text { Cantidad de participantes en proyectos de } \\
\text { recuperación de ecosistemas estratégicos } \\
\text { Número de personas de la localidad que participan } \\
\text { en actividades educativas ambientales } \\
\text { Participación de grupos locales en actividades de } \\
\text { protección de espacios naturales } \\
\text { Número y frecuencia de intercambio de experiencias } \\
\text { y diálogo de saberes en relación con dinámicas } \\
\text { ambientales locales }\end{array}$ & & & & \\
\hline
\end{tabular}




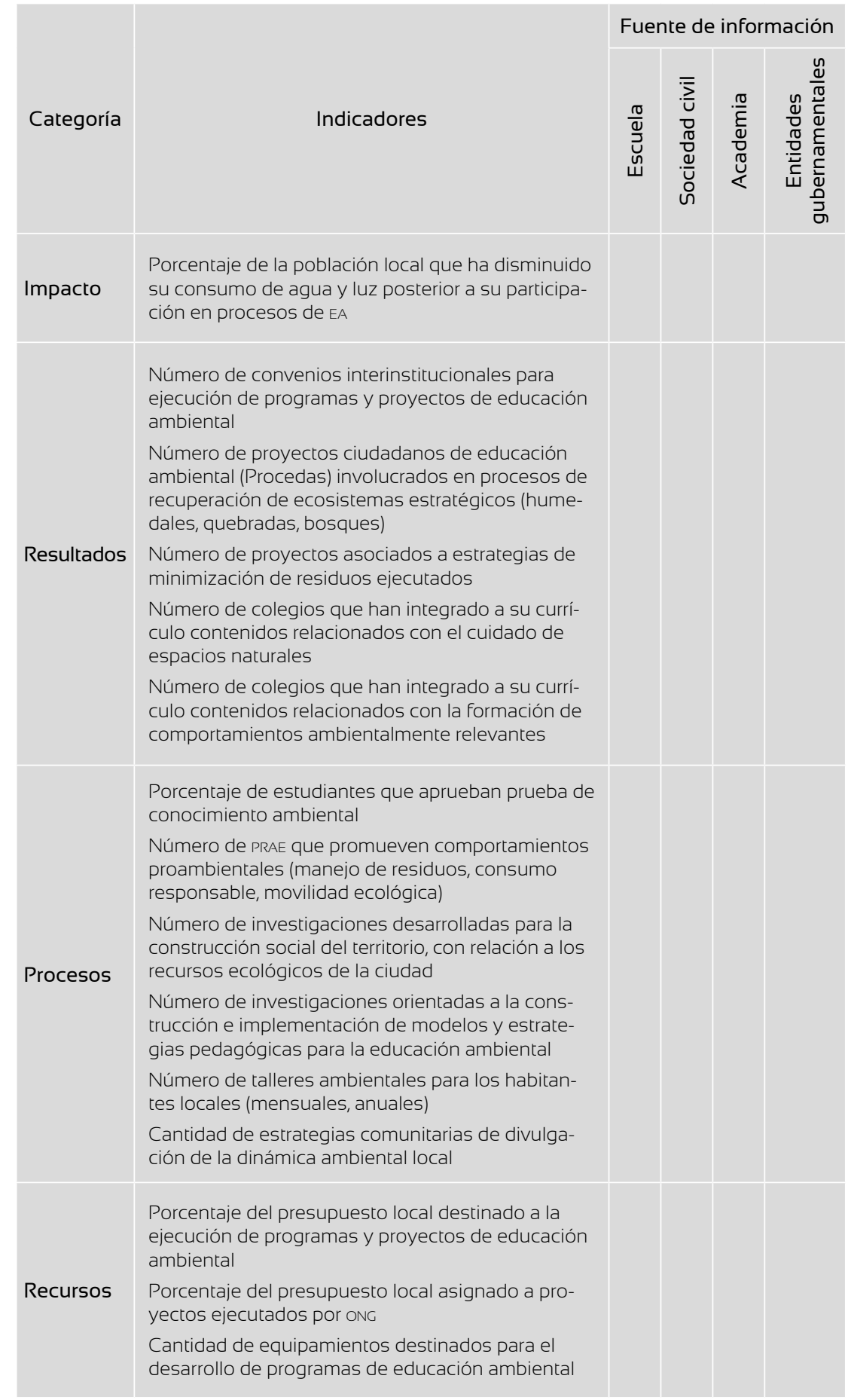




\section{Conclusiones}

El gran progreso en materia de evaluación del estado de los recursos ecológicos y el alto grado de avance conceptual en materia de educación ambiental no se corresponden con el desarrollo de indicadores que permitan evaluar el impacto de las actividades, los proyectos y programas adelantados de manera prolífica en la educación ambiental desde diferentes frentes, como la escuela, las organizaciones sociales, el ámbito académico y el sector gubernamental. Por esta razón, se desconoce su impacto en torno a la conservación de los recursos ecológicos, a la defensa del patrimonio ambiental del país y principalmente a la formación de conciencia ambiental en las personas y a la transformación de los modos de actuar entre los individuos y de estos con su entorno (Burbano, 2009).

Estamos en la era de la educación basada en la evidencia (Páramo, 2017). Como afirma el autor, la sociedad espera que las políticas educativas que se diseñen en los programas de gobierno, incluyendo desde luego la política ambiental, tengan resultados favorables de mejoramiento ambiental y principalmente en nuestra manera de relacionarnos con el entorno próximo. A este respecto surgen interrogantes del tipo: ¿Qué tan efectivos son los procesos de educación ambiental que se implementan en los distintos entornos de aprendizaje para formar a las personas? ¿Cuál es el panorama de las investigaciones en educación ambiental en nuestro país? ¿Cuál es el horizonte hacia donde deben dirigirse los esfuerzos en materia de educación ambiental? ¿Cuáles estrategias de educación ambiental han sido más significativas? Para ello se requieren pruebas. La medición de los procesos a través de indicadores puede proveer dichas evidencias.

En este artículo se ha presentado una propuesta de organización de un sistema de indicadores para monitorear la educación ambiental en Colombia, que incluye pautas operativas para la recolección de información de los procesos de educación ambiental, una estructura conformada por dos dimensiones en torno al eje de comportamientos ambientalmente relevantes y el enunciado de un grupo de indicadores para cada categoría de información, que se constituyan en el punto de partida de una batería de indicadores de educación ambiental. Con esto esperamos contribuir a configurar un panorama objetivo del desarrollo de la educación ambiental en nuestro país, porque consideramos que, gracias a los indicadores, se puede proyectar la educación ambiental en sus distintos niveles de actuación y se podrá ir consolidando conocimiento alrededor de aquellas acciones que resultan más eficaces. 
El estudio de los indicadores y sus relaciones emerge entonces como un reto de la investigación en el campo de la educación ambiental para fortalecer el impacto en la contextualización de sus acciones y de sus proyecciones (Torres, 2012) y como herramienta clave para la toma de decisiones y el diseño de políticas públicas en educación.

\section{Referencias}

Bennet, D. (1993). Evaluación de un programa de educación ambiental. Serie de Educación Ambiental, n. ${ }^{\circ}$ 12. Biblioteca deunesCo-Pnuma.

Burbano, A. M. (2009). La convivencia ciudadana: su análisis a partir del "aprendizaje por reglas". Revista Colombiana de Educación, 57, 28-45.

Chacón, R. (2004). La calidad de vida y la planificación urbana. Departamento de Planificación Urbana, Universidad Simón Bolívar, Caracas.

Comisión Económica para América Latina y el Caribe, Cepal. (2007). Indicadores ambientales y de desarrollo sostenible: Avances y perspectivas para América Latina y el Caribe. Serie Manuales 55. Santiago de Chile.

Corral, V. (2000). La definición del comportamiento proambiental. La psicología social en Mexico, 8, 466-472.

Corral, V. y Pinheiro, J. (2004). Aproximaciones al estudio de la conducta sustentable. Medio Ambiente y Comportamiento Humano, 5, 1-26.

De Esteban, G. (2002). Análisis de indicadores de desarrollo de la educación ambiental en España. Ecosistemas, 11(1).

De Esteban, G; Benayas, J. y Gutiérrez, J. (2000). La utilización de indicadores de desarrollo de la educación ambiental como instrumento para la evaluación de políticas de educación ambiental. Tópicos en Educación Ambiental, 2, 61-72.

Departamento Administrativo Nacional de Estadística, DANE. (2012). Guía para diseño, construcción e interpretación de indicadores. Estrategia para el fortalecimiento estadístico territorial.

García, D. y Benayas, J. (2007). Indicadores de educación ambiental en municipios: diseño y relaciones con otros Indicadores de sostenibilidad. Comunicación presentada en la I International Conference on Sustainibility Measurement and Modelling. Terrassa, Barcelona

Hederich, C.; Martínez, J. y Rincón, L. (2014). Hacia una educación basada en la evidencia. Revista Colombiana de Educación, 66, 19-54.

Heras, F. y Sintes, M. (2004). Evaluación de actuaciones de educación, comunicación y sensibilización en materia de residuos (p. 192). Madrid: Ceneam-OAPN (Ministerio de Medio Ambiente).

Hungerford, H. R. y Litherland, R. (1986). Módulos de acción ambiental. Illinois: Stripe. 
Leva, G. (2005). Indicadores de calidad de vida urbana. Teoría y metodología. Buenos Aires: Politike.

Manteiga, L. (2000). Los indicadores ambientales como instrumen t o para el desarrollo de la política ambiental y su integración en otras políticas. Estadística y Medio Ambiente, 75-87.

Mayer, M. (2006). Criterios de calidad e indicadores en educación ambiental. Perspectivas internacionales y ejemplos nacionales e internacionales a la vista de la Década de las Naciones Unidas de la Educación para el Desarrollo Sostenible. Zaragoza.

Medina, I. F. y Páramo, P. (2014). La investigación en educación ambiental en América Latina: un análisis bibliométrico. Revista Colombiana de Educación, 66, 55-72.

Ministerio de Ambiente, Vivienda y Desarrollo Territorial, MAVDT (2007). Marco conceptual del Sistema de Información Ambiental de Colombia-SIAC. Bogotá: autor.

Ministerio de Educación Nacional. (2013). Sistema Nacional de Indicadores Educativos para los niveles de preescolar, básica y media en Colombia. Bogotá: autor.

Ministerio de Educación Nacional. Colombia. Web site: http://www.mineducacion.gov.co

Ministerio del Medio Ambiente de Colombia. (2002). Sistema de Indicadores de Sostenibilidad Ambiental en el Ambito Nacional-avances y perspectivas. Recuperado de http://www.minambiente.gov.co/sisa/ capitulos_1_4/cap_2/cap2a.htm.

Observatorio Ambiental de Bogotá. Sitio web: http://oab.ambientebogota.gov.co/

Organización de las Naciones Unidas para la Educación, la Ciencia y la Cultura. (2011). Education for sustainable development. An expert review of processes and learning. París, Francia.

Páramo, P. (2009). Pedagogía urbana: elementos para su delimitación como campo de conocimiento. Revista Colombiana de Educación, 57, 14-27.

Páramo, P. (2010). Aprendizaje situado: creación y modificación de prácticas culturales en el espacio público urbano. Psicología y Sociedad, 22(1), 130-138.

Páramo, P. (en prensa). La evidencia en la pedagogía y en las políticas públicas de educación. Revista de la Facultad de Medicina. Universidad Nacional de Colombia.

Páramo, P. y Hederich, C. (2014). Educación basada en la evidencia. Revista Colombiana de Educación, 66, 13-16.

Quiroga, R. (2007). Propuesta regional de indicadores complementarios al Objetivo de Desarrollo del Milenio 7. Serie Estudios Estadísticos y Prospectivos Comisión Económica para América Latina y el Caribe. Santiago, Chile. 
Ramírez, L. (2002). Indicadores ambientales. Situación actual y perspectivas. Serie Técnica. Madrid: Ministerio de Medio Ambiente.

Rey, C. (2002). Indicadores de sostenibilidad ambiental. Observatorio Medioambiental (vol. 5, pp. 79-99). S. d.

Secretaría Distrital de Ambiente. (2015). Recuperado de http://www.ambientebogota.gov.co/.

Secretaría Distrital de Gobierno de Bogotá. Sitio web: http://www.gobiernobogota.gov.co/

Stubelj, A. (2013). Assessment of environmental education indicators in Triglav National Park, Slovenia (pp. 751-754). S. d.

Suárez, D. C. (2003). Indicadores e Índices Ambientales: Marco Teórico de Indicadores. Programa de Información e Indicadores de Gestión de Riesgos de Desastres Naturales. Ejecución del componente II. Indicadores de Gestión de Riesgos. BID-Cepal-IDEA. Manizales, Colombia.

Sureda, J.; Oliver, M. y Castells, M. (2004). La evaluación de la educación, la interpretación y la información ambiental en espacios naturales protegidos. Diseño de un modelo. Seminario Equipamientos de Educación Ambiental, Ceneam 7-8 octubre 2004.

Torres, M. (2012). La política nacional de educación ambiental en Colombia: un marco para la exploración y la reflexión, sobre las necesidades investigativas en educación ambiental. En Investigación y educación ambiental. Apuestas investigativas pertinentes a los campos de reflexión e intervención en educación ambiental [edición especial en celebración de los 10 años de la Política Nacional de Educación Ambiental 2002]. MEN-Corantioquia.

Wilches, G. (2008). Brújula, bastón y lámpara para trasegar los caminos de la educación ambiental. Bogotá: Ministerio de Ambiente, Vivienda y Desarrollo Territorial. 